\title{
Calculation of Temperature Rise of the High-voltage Winding in the Power Transformer under DC Magnetic Biasing
}

\author{
Yunyan XIA ${ }^{1,2, a, *}$, Yongming $\mathrm{XU}^{1, \mathrm{~b}}$ and Yongsen $\mathrm{HAN}^{1, \mathrm{c}}$ \\ ${ }^{1}$ Harbin University of Science and Technology, Harbin, Heilongjiang 150080, China. \\ ${ }^{2}$ Jiangsu Huapeng Group Co. Ltd, Liyang, Jiangsu 213300, China. \\ a yunyan_x@163.com, b327325977@qq.com, c hys2006@hrbust.edu.cn \\ *corresponding author
}

Keywords: Temperature rise, Power transformer, DC magnetic biasing

\begin{abstract}
The larger power transformers are greatly affected by the DC magnetic biasing phenomenon, which may cause the local overheating and threaten the safe operation of the power transformer. We aimed at studying the temperature distribution and temperature rise in the power transformer under DC magnetic biasing current. A $240000 \mathrm{kVA} / 330 \mathrm{kA}$ oil immersible power transformer is studied. The 3D model of the power transformer and the DC magnetic biasing injection are established, respectively. According to the calculation of fluid field and the highvoltage winding loss, the temperature distributions of the power transformer are obtained. The results show that the temperature rise and the high-voltage winding loss are greatly affected by the DC magnetic biasing phenomenon.
\end{abstract}

\section{Introduction}

DC magnetic biasing phenomenon may caused by a geomagnetic storm or the injection of DC current into ac power transformers, accelerating the power transformer core saturation and causing the local overheating or vibration [1,2]. The high-altitude nuclear electromagnetic pulse generated in the geomagnetic storm will induced the geomagnetically induce current in power transformer. DC current in HVDC power transmission over long distance can flow in ac power transformers. DC magnetic biasing phenomenon seriously threatens the safe operation of power transformer and has attracted broad attention.

The DC magnetic biasing phenomenon caused by DC current injection has been already studied for several decades. The magnetization characteristic of transformer laminated core is analyzed under the DC magnetic biasing [3]. The effect and its suppression method of DC magnetic biasing on the relay protection equipment in power system and transmission system operation are also considered [4]. In addition, finite-element simulations combined analytical studies were carried out to determine a tolerable dc current limit of power transformer under DC magnetic biasing, and the tie plates of core transformer are the components most susceptible to rapid temperature rise [5]. However, very few studies have been carried out on the temperature rise of transformer winding caused by the DC magnetic biasing.

In this paper, a 3D model of $240000 \mathrm{kVA} / 330 \mathrm{kA}$ oil immersible power transformer is established and the high-voltage winding loss of this power transformer is then calculated under different DC magnetic biasing current. According to the high-voltage winding loss and the calculation of fluid field in this power transformer, the temperature distributions of the studied power transformer are obtained by considering the effect of DC magnetic biasing current. This study provides theoretical basis for the optimization design of winding insulation structure of highvoltage and high-capacity power transformer.

\section{Model of Power Transformer}

A $240000 \mathrm{kVA} / 330 \mathrm{kA}$ oil immersible power transformer is taken as an example, which is a 
three-phase winding transformer. The winding is divided into primary and secondary windings. Considering the effect of side yoke, the magnetic field distribution of this power transformer is different from the three-limb transformer, so it is not reasonable to only study one phase. The three dimensional finite element model of the studied power transformer is built, as shown Figure 1, consisting of core, three-phase winding and iron core structure. The basic parameters of the windings are shown in Table 1.

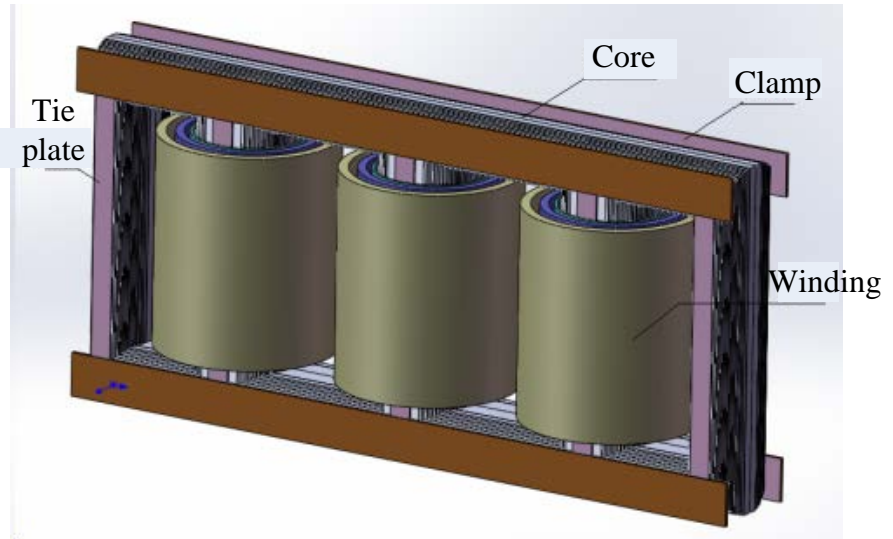

Figure 1 3D model of power transformer.

Table 1 Basic parameters of transformer windings.

\begin{tabular}{|c|c|c|c|}
\hline Parameters & Inner diameter $(\mathrm{mm})$ & $\begin{array}{c}\text { External diameter } \\
(\mathrm{mm})\end{array}$ & Height (mm) \\
\hline Low-voltage winding & 1014 & 1063 & 2000 \\
\hline $\begin{array}{c}\text { Medium-voltage } \\
\text { winding }\end{array}$ & 1167 & 1321 & 2000 \\
\hline High-voltage winding & 1483 & 1669 & 2000 \\
\hline
\end{tabular}

\section{DC Magnetic Biasing Injection in Power Transformer}

To calculate the high-voltage winding loss of power transformer under different DC magnetic biasing current, the field-circuit coupling method is used to establish the external circuit model of the power transformer, as shown in Figure 2.The established circuit model is used as the external excitation source for the finite element model of power transformer. The high-voltage winding loss under different DC magnetic biasing current can be achieved by adjusting the DC current source in the external circuit model as shown in Figure 2. 


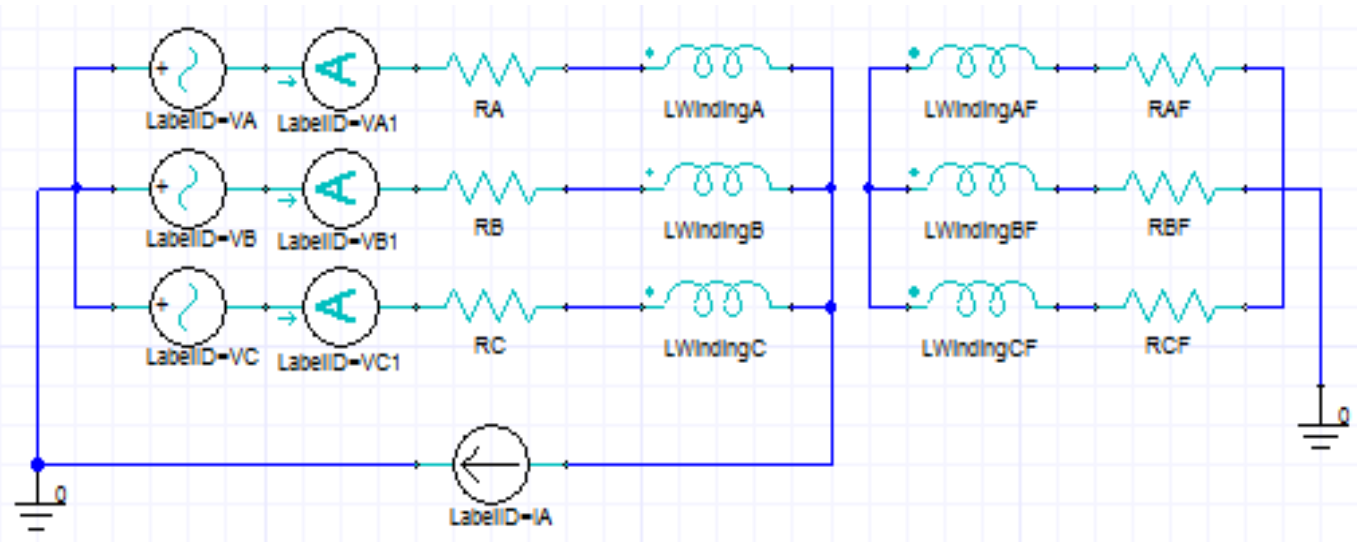

Figure 2 External circuit model of the power transformer. Where LabellD=VA, LabellD=VB and

LabellD $=$ VC are three-phase voltage sources, respectively. LabellD=VA1, LabellD=VB1 and

LabellD=VC1 are amperemeter. LWindingA, LWindingB and LWindingC are primary induction coils. LWindingAF, LWindingBF and LWindingCF and secondary induction coils. RA, RB and RC are the equivalent resistance of high-voltage windings and the resistance value is $3.15 \Omega$. RAF, RBF and RCF are the equivalent resistance of low-voltage windings and the resistance value is $1.56 \mathrm{~m} \Omega$.

LabellD = IA is DC current source for generating different DC magnetic biasing current.

\section{Fluid field in Power Transformer}

According to the internal fluid flow path and concrete structure of the power transformer, the whole fluid field of the power transformer as shown in Figure 3 can be divided into the winding oil circuit, the cooler and the oil circuit in the connecting structure, etc. By the analysis of the fluid field, the flow resistance is obtained and then is lined up via fluid network to calculate the actual flow of oil circuit. The finite element method combined with the fluid network is used to calculate the fluid field and the flow resistance.

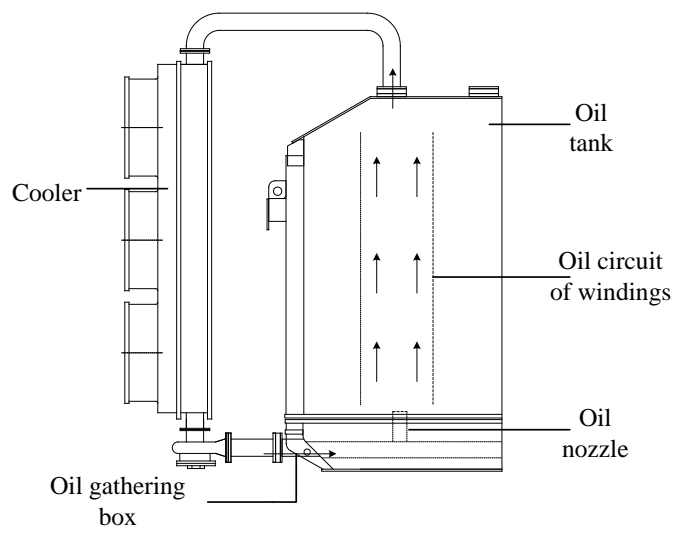

Figure 3 Whole fluid field of the power transformer

The flow resistance $(h)$ can be obtained according to the pressure difference between the inlet and outlet $(\Delta p)$ and the corresponding flow conditions $(Q)$, as below,

$$
h=\Delta p / Q
$$

Based on the flow resistance, the percentage rate $\left(P_{e x}\right)$ for the actual flow quantity of each windings as Table 2 can be calculated as follows for determining the temperature distribution in the power transformer.

$$
P_{e x}=\frac{1 / h_{x}}{1 / h_{\mathrm{h}}+1 / h_{\mathrm{m}}+1 / h_{1}+1 / h_{\mathrm{r}}}
$$

Table 2 Percentage rate for the actual flow quantity of each windings. 


\begin{tabular}{|c|c|}
\hline Type & Percentage rate (\%) \\
\hline Low-voltage winding $\left(h_{l}\right)$ & 16.10 \\
\hline Regulating winding $\left(h_{r}\right)$ & 21.60 \\
\hline Medium-voltage winding $\left(h_{m}\right)$ & 28.03 \\
\hline
\end{tabular}

\section{Effect of DC Magnetic Biasing on the Transformer Loss}

The transient field-circuit coupling finite element method is used to calculate the magnetic field distribution in the high-voltage windings under DC magnetic biasing, and the electromagnetic phasor in the power transformer satisfies,

$$
\begin{array}{r}
\nabla \times v \nabla \times A=J_{D C}+J_{C}+\sigma \frac{\partial A}{\partial t} \\
i_{1} R+L_{1} \frac{d i_{1}}{d t}+e=V_{1}
\end{array}
$$

where $L_{1}$ is the sum of the lead inductance and the source inductance of power tansformer. $R$ is the sum of the lead resistance and the source resistance. $i_{1}$ is the primary excitation current. $v$ is magnetic reluctivity. $A$ is the magnetic vector. $J_{D C}$ is the DC magnetic biasing current density. $J_{C}$ is the AC excitation current density. $\sigma$ is the conductivity.

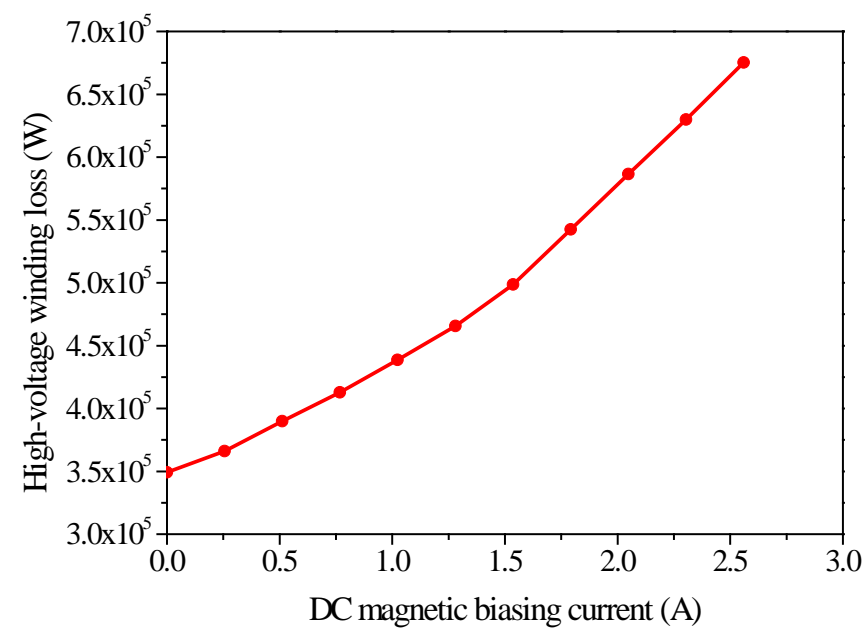

Figure 4 High-voltage winding losses under DC magnetic biasing.

The high-voltage winding loss of power transformer consists of eddy-current loss and resistance loss. The internal magnetic density distribution of power transformer can be calculated by using Eqs. 2 and 3 to obtain the eddy-current loss density of high-voltage winding. According to the loss density, the eddy-current loss in each winding is calculated from the integration along the axial direction of the winding. The resistance loss of high-voltage winding can be obtained based on $P_{R}=I^{2} R$.

The high-voltage winding losses under DC magnetic biasing are then calculated, as shown in Figure 4. It can be seen from Figure 4 that, with increasing the DC magnetic biasing current, the high-voltage winding loss is also increased. This is attributed to the fact that the increasing DC magnetic biasing current accelerating the core saturation and aggravating the distortion of the winding exciting current.

\section{Effect of DC Magnetic Biasing on the Temperature Rise}

The high voltage winding losses under different DC magnetic biasing current are considered as the heat source for calculating the temperature field in the high voltage winding. The temperature field analysis is based on the fluid network. The above obtained flow characteristics of transformer oil implements the whole series of temperature field. 
The entrance oil temperature is $298 \mathrm{~K}$ and is only used for calculating the convective heat transfer coefficient which is modified during the temperature distribution calculation. The winding oil velocity is $0.35 \mathrm{~m} / \mathrm{s}$, and the oil flow is $14.26 \mathrm{~kg} / \mathrm{s}$. The two sides of the model are symmetry boundary conditions, namely the geometric and physical properties on both sides of the boundary is symmetrical along the cross section.

The temperature distribution of single section high-voltage winding under DC magnetic biasing current with value of $2.56 \mathrm{~A}$ has been exhibited in Fig. 5. It can be seen that the temperature in parts of the high-voltage winding exceeds $99{ }^{\circ} \mathrm{C}$, especially in the top and middle windings. The above temperature exceeds the maximum allowable temperature of $98{ }^{\circ} \mathrm{C}$, seriously threatening the safe operation of power transformer.

The temperature rise of Point $P$ (as shown in Figure 5) in the high-voltage winding under different DC magnetic biasing current can be obtained as shown in Figure 6 by repeating the same calculation procedure for the temperature distribution of the power transformer.

It can be observed from Figure 6 that the temperature rise of the high-voltage winding is gradually increased with the increasing DC magnetic biasing current. The temperature rise exceeds the allowable temperature limitation of $65 \mathrm{~K}$ proposed in the national standard when the DC magnetic biasing current is larger than $2.0 \mathrm{~A}$. Thus, the local overheating caused by DC magnetic biasing should gain more attention and the suppression method for the DC magnetic biasing should further be studied.

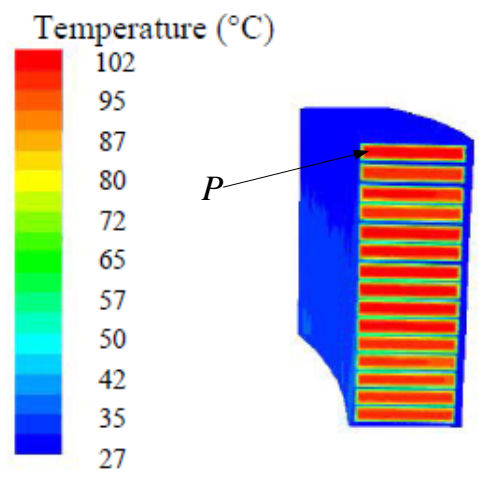

Figure 5 Temperature distribution in one section high-voltage winding under DC magnetic biasing.

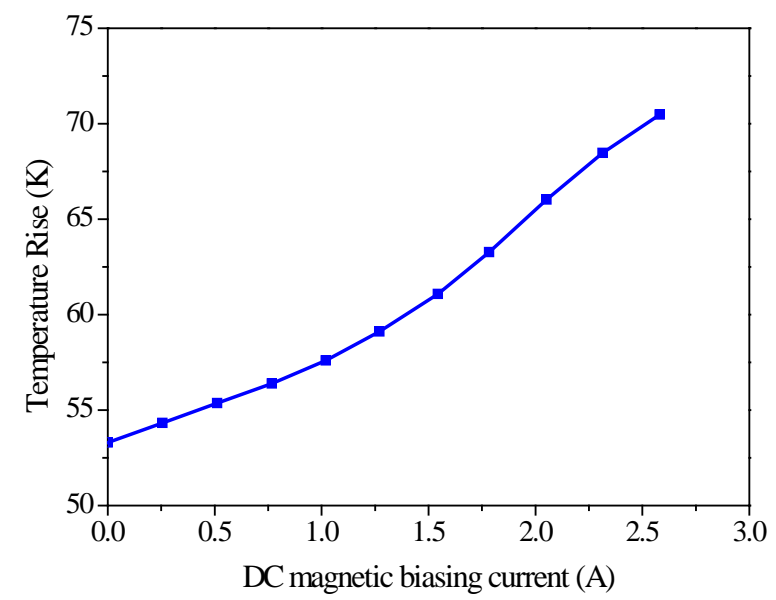

Figure 6 Temperature rise in the high-voltage winding under different DC magnetic biasing current.

\section{Conclusion}

The field-circuit coupling method is used to calculate the winding loss and temperature rise of a $240000 \mathrm{kVA} / 330 \mathrm{kA}$ oil immersible power transformer. The winding loss of high-voltage winding is increased with the increasing the applied DC magnetic biasing current, and the transformer core appears the saturation or over-saturation. The temperature rise of the high-voltage winding is gradually increased with the increasing DC magnetic biasing current, may cause the local over- 
heating in the high-voltage windings.

\section{References}

[1] Yang X., Wang F., Duan R., Zheng H. (2014) Simulation study of DC bias enduring ability of $500 \mathrm{kV}$ power transformer. Power system protection and control, 42, 60-66.

[2] Price P.R. (2002) Geomagnetically induced current effects on transformers. IEEE Trans Power Delivery, 7, 1002-1008.

[3] Andreas D., John M., Thomas Z. (2009) Three-phase transformer model including magnetic hysteresis and eddy currents effects. IEEE Trans Power Delivery, 24, 1284-1194.

[4] Li H., Cui X., Liu T., Cheng Z., Liu D., Jiao C. (2009) Electric circuit and magnetic circuit combined model of DC biased power transformer. Proceedings of the CSEE, 29, 119-125.

[5] Picher P., Bolduc L., Dutil A., Pham V. (1997) Study of the acceptable DC current limit in coreform power transformers, IEEE Trans Power Delivery, 12, 257-265. 